\title{
ON THE CLOSED GRAPH THEOREM ${ }^{1}$
}

\section{ALAN McINTOSH}

1. Introduction. In this paper several versions of the closed graph theorem are presented. That is, we give various conditions on pairs $(E, F)$ of locally convex linear topological spaces which ensure that every closed linear map $T$ of $E$ into $F$ is continuous. We do not explicitly consider additional restraints (such as almost continuity) on $T$.

It is known that the closed graph theorem holds if $E$ is barreled and $F$ is $\left(B_{r}\right)$-complete (see [6] or [7, IV, 8.5]). We define a class of spaces (called $\left(C_{r}\right)$-spaces) which is larger than the class of $\left(B_{r}\right)$ complete spaces, and show that the closed graph theorem still holds if $E$ is barreled and $F$ is a $\left(C_{r}\right)$-space (Theorem 1(i)). This is achieved by looking at the duals $E^{\prime}$ and $F^{\prime}$ under their weak topologies, and showing that the dual map $T^{\prime}$ has domain $F^{\prime}$.

By considering the duals of $E$ and $F$ under their Mackey or strong topologies, we are led to several other versions of the closed graph theorem. The more explicit of these results are collected in Theorem 1 , while the general results needed in their proofs are scattered through the paper. Of course, when investigating a particular pair of spaces $(E, F)$, we may be able to apply one of the general theorems when Theorem 1 fails.

We will just mention one further form of the closed graph theorem here. Let $E$ be a sequentially complete Mackey space with complete strong dual, and let $F$ be a $(F)$-, a $(L F)$ - or a suslinian 1.c.s. Then the closed graph theorem is known to hold for the pair $(E, F)$ if $E$ is bornological (Theorem 1(iv)). We prove in Theorem 1 (v) that if $F$ is reflexive, then the closed graph theorem holds even if $E$ is not bornological. Since the distribution spaces are all reflexive suslinian 1.c.s.'s [8], Theorem $1(\mathrm{v})$ gives a generalization of Schwartz' result [8] that the closed graph theorem holds for the pair $(E, F)$ if $E$ is sequentially complete bornological and $F$ is one of the distribution spaces.

We use the notation and terminology of Schaefer [7]. By a 1.c.s., we mean a (real or complex) locally convex linear topological space, always assumed Hausdorff. All topologies mentioned are assumed to be locally convex. If $E$ is a l.c.s., then $\sigma, \tau$ and $\beta$ denote the weak,

Received by the editors October 6, 1967.

1 This research was supported by a grant from the Air Force Office of Scientific Research, Grant AF-AFOSR-1071-66. 
Mackey and strong topologies on $E$ and $E^{\prime}$ with respect to the duality $\left\langle E, E^{\prime}\right\rangle$ (where $E^{\prime}$ is the topological dual of $E$ ). The topology $\sigma\left(E^{\prime}, E\right.$ ) on $E^{\prime}$ is called the weak (rather than weak ${ }^{*}$ ) topology. A 1.c.s. $(E, J)$ is defined to be a Mackey space if $J=\tau\left(E, E^{\prime}\right)$. If $B$ is a subset of $E$ (or $E^{\prime}$ ), then $B^{-}$denotes its closure and $B_{\sigma}^{-}$its $\sigma$-closure. If $T$ is a map from $E$ to $F$, then $D(T)$ denotes the domain of $T$. We say that $T$ is a map of $E$ into $F$ if $D(T)=E$.

2. For easy reference, we present the more explicit results of the paper in the following theorem. The $\left(C_{r}\right)$-spaces are defined in $\$ 3$, while ultra-bornological and suslinian 1.c.s.'s are defined in $\$ 7$. For convenience, we let $\mathfrak{C}$ denote the family of pairs of 1.c.s.'s for which the closed graph theorem holds. That is, we write $(E, F) \in \mathcal{C}$ if every closed linear map of $E$ into $F$ is continuous.

THEOREM 1. Let $E$ and $F$ be two locally convex spaces. Then $(E, F) \in \mathbb{e}$ if $E$ is a Mackey space, and one of the following eight conditions is satisfied:

(i) $E$ is barreled and $F$ is a $\left(C_{r}\right)$-space;

(ii) $E_{\sigma}^{\prime}$ is complete;

(iii) $E_{\tau}^{\prime}$ is sequentially complete and $F_{\tau}^{\prime}$ is metrizable;

(iv) $E$ is ultra-bornological and $F$ satisfies one of the following: $(\alpha)$ $F$ is a $\left(C_{r}\right)$-space; $(\beta) F$ is suslinian; or $(\gamma)$ the topology $S$ on $F$ is the inductive topology with respect to a sequence $\left(F_{n}, h_{n}\right)$ of $(B)$-complete spaces such that $F=U h_{n}\left(F_{n}\right)$ (e.g. $F$ is an $(L F)$-space);

(v) $E$ is sequentially complete, $E_{\beta}^{\prime}$ is complete, and $F$ is a semireflexive space satisfying $(\alpha),(\beta)$ or $(\gamma) ;^{2}$

(vi) $E$ is sequentially complete, $\left(E^{\prime}, J\right)$ is quasi-complete for some $J$ such that $\sigma \subseteq J \subseteq \beta$, and $F$ is a semi-reflexive $\left(C_{r}\right)$-space;

(vii) $E$ is sequentially complete, $\left(E^{\prime}, J\right)$ is sequentially complete for some $J$ such that $\sigma \subseteq J \subseteq \beta$ and $F$ is a semireflexive space with metrizable dual $F_{\beta}^{\prime}$;

(viii) $F_{\sigma}$ is complete.

REMARK. Part (iii) could be stated and proved with $E_{\tau}^{\prime}$ replaced by " $\left(E^{\prime}, J\right)$ for some $J$ such that $\sigma \subseteq J \subseteq \tau$ ". But this would not really be a stronger result, for it can be shown that if $\left(E^{\prime}, J\right)$ is sequentially complete, then $E_{r}^{\prime}$ has the same property. Similarly, (v) could be stated and proved with " $E_{\tau}^{\prime}$ " replaced by " $\left(E^{\prime}, J\right)$ for some $J$ such that $\sigma \subseteq J \subseteq \beta^{\prime \prime}$. But it is known that $E_{\beta}^{\prime}$ is complete if $\left(E^{\prime}, J\right)$ is complete $[3,18.3]$.

2 In parts (iv) and (v) we can add: $(\delta) F$ is a member of the class $D$ defined by Raikov (Sibirsk Mat. Ž. 7 (1966), 353-372). 
The proof of Theorem 1 is contained in $\$ \$ 5-8$.

3. By analogy with the $(B)$-complete and $\left(B_{r}\right)$-complete spaces of Ptak, we define $(C)$-spaces and $\left(C_{r}\right)$-spaces as follows. ${ }^{3}$

Definition. A l.c.s. $F$ is a $(C)$-space (resp. $\left(C_{r}\right)$-space) if every linear subspace (resp. dense linear subspace) $D$ of $F_{\sigma}^{\prime}$ whose intersection with each $\sigma$-bounded subset $B$ of $F^{\prime}$ is $\sigma$-closed in $B$, is necessarily closed in $F_{\sigma}^{\prime}$.

It is clear that every $(C)$-space is a $\left(C_{r}\right)$-space. Also, every $(B)$ complete (resp. $\left(B_{r}\right)$-complete) space is a $(C)$-space (resp. $\left(C_{r}\right)$-space). If $F$ is barreled, then the $\sigma$-bounded subsets of $F^{\prime}$ are precisely the equicontinuous sets $[7, \mathrm{IV}, 5.2]$. So a barreled $(C)$-space is $(B)$ complete, and a barreled $\left(C_{r}\right)$-space is $\left(B_{r}\right)$-complete.

In the definition we could require that the sets $B$ be convex. So the definition is not altered if the $\sigma$-topology on $F^{\prime}$ is replaced by the Mackey topology.

Examples. 1. If $F$ is a Fréchet space, then both $F$ and $F_{\tau}^{\prime}$ are $(B)$-complete and hence $(C)$-spaces. For a proof of this, and for other facts about $(B)$-complete and $\left(B_{r}\right)$-complete spaces, see $[7$, p. 162] (or [3], problem 18I). Note that a $\left(B_{r}\right)$-complete space is complete.

2. If $(F, J)$ is a $(C)$-space or a $\left(C_{r}\right)$-space, then so is $\left(F, J_{1}\right)$, where $J_{1}$ is any topology consistent with the duality $\left\langle F, F^{\prime}\right\rangle$. If $E$ is an infinite dimensional Fréchet space, then $E_{\sigma}^{\prime}$ is an example of a $(C)$-space that is not $\left(B_{r}\right)$-complete (since $E_{\sigma}^{\prime}$ is not complete [7, p. 148]).

3. If $F_{\tau}^{\prime}$ is metrizable, then $F$ is a $(C)$-space. Note that $F$ need not be complete even under its Mackey topology (e.g. let $F$ be the space $E_{\tau}^{\prime}$ in problem $18 \mathrm{~F}$ of $\left.[3]\right)$. So even a Mackey $(C)$-space need not be $\left(B_{r}\right)$-complete.

4. Let $E$ be a closed subspace of the 1.c.s. $F$. If $F$ is a $(C)$-space or a $\left(C_{r}\right)$-space, then $E$ (under the induced topology) has the same property. This can be proved by adapting the proof for $(B)$ - and $\left(B_{\boldsymbol{r}}\right)$ complete spaces in $[7, I V, 8.2]$. If $F$ is a $(C)$-space, then so is $F / E$ (under the quotient topology). This result can be proved by direct verification.

5. Husain [2] defines several spaces that are related to the closed graph theorem. In particular, we note that every $(C)$-space $\left(\left(C_{r}\right)\right.$ space) is a $B(g)$-space $\left(B_{r}(g)\right.$-space) (by Lemma 1 of Chapter $7, \S 6$ in [2]). Let $E_{\omega}$ denote an infinite dimensional Banach space $E$ with the finest locally convex topology $\omega$. Husain notes ([2], Chapter 7, 81) that $E_{\omega}$ is not a $B_{r}(\mathcal{g})$-space. So $E_{\omega}$ is an example of a complete

${ }^{3}$ In a recent paper (Math. Scand. 19 (1966), 54-58), A. Persson has also introduced $(C)$ - and $\left(C_{r}\right)$-spaces (using the names $t$-polar and weakly $t$-polar spaces), and has proved Theorem 1 (i) and the equivalence of (i), (ii) and (iii) in Theorem 2(b). 
barreled space that is not a $\left(C_{r}\right)$-space. Note that the identity map of $E$ into $E_{\omega}$ is an example of a closed linear map of a Banach space into a complete barreled space that is not continuous.

6. The main questions left unanswered are those which ask whether certain spaces or classes of spaces are $\left(C_{r}\right)$-spaces. For example, is $D$ or $D^{\prime}$ a $\left(C_{r}\right)$-space? Note that an $(L F)$-space is not necessarily a $(C)$-space (see [3], problem $22 \mathrm{C}$ ), and the strong dual of a reflexive $(L F)$-space is not necessarily a $(C)$-space (see [9], p. 93). Is every $\left(C_{r}\right)$-space a $(C)$-space?

4. The following two lemmas will be used repeatedly.

Lemma 1. Let $T$ be a closed linear map of a Mackey space $E$ into a l.c.s. F. Then its dual $T^{\prime}$ is a closed continuous linear map from $F_{\sigma}^{\prime}$ to $E_{\sigma}^{\prime}$ with domain $D\left(T^{\prime}\right)$ dense in $F_{\sigma}^{\prime}$. If $D\left(T^{\prime}\right)=F^{\prime}$, then $T$ is continuous.

Proof. It is well known that $T^{\prime}$ is closed and densely-defined, and it is readily shown that $T^{\prime}$ is continuous. For the final result, see $[7, \mathrm{IV}, 7.4]$.

When proving that $T^{\prime}$ is defined everywhere, we will apply the following lemma.

Lemma 2. Let $S$ be a closed continuous densely-defined linear map from a l.c.s. $G$ to a l.c.s. $H$. Then $D(S)=G$ if one of the following conditions is satisfied:

(a) $H$ is complete;

(b) $H$ is quasi-complete and $G$ is the weak or Mackey dual of $a\left(C_{r}\right)$ space;

(c) $H$ is sequentially complete and $G$ is metrizable.

Proof. (a) Let $u \in G$. Let $\left\{u_{\alpha}\right\}$ be a net, consisting of elements of $D(S)$, and converging to $u$. Then $\left\{S u_{\alpha}\right\}$ is a Cauchy net in $H$ which must converge to an element $v$ of $H$. So, since $S$ is closed, $u \in D(S)$.

(b) It suffices to prove that, for each bounded subset $B$ of $G$, $D(S) \cap B$ is closed in $B$. Let $\left\{u_{\alpha}\right\}$ be a net in $D(S) \cap B$ converging to an element $u \in B$. We must prove that $u \in D(S)$. Now $\left\{S u_{\alpha}\right\}$ is a bounded Cauchy net in $H$, which, by the quasi-completeness of $H$, must converge to an element $v$. We now apply the fact that $S$ is closed to conclude that $u \in D(S)$.

(c) is proved in the same way as (a), except that we consider a sequence $\left\{u_{n}\right\}$ instead of the net $\left\{u_{\alpha}\right\}$.

5. We are now in a position to prove parts (i) and (ii) of Theorem 1. (Note that $E$ is barreled if and only if $E$ is a Mackey space with quasi-complete weak dual $[4, \$ 23.6(4)]$.) 
Proof of (i). Let $T$ be a closed linear map of $E$ into $F$. Then $T^{\prime}$ is a closed continuous and densely defined map from $F_{\sigma}^{\prime}$ to $E_{\sigma}^{\prime}$. Apply Lemma 2 (b) to see that $D\left(T^{\prime}\right)=F^{\prime}$, and Lemma 1 to conclude that $T$ is continuous.

Proof of (ii). The proof is the same, except that we apply Lemma 2(a) instead of Lemma 2(b).

Theorem 1(i) has two partial converses that should be taken note of when looking for new versions of the closed graph theorem. We state them in the following theorem.

Theorem 2. (a) A l.c.s. $E$ is barreled iff $(E, G) \in \mathcal{e}$ for all Banach spaces $G$.

(b) Let $(F, J)$ be a barreled space. Then the following conditions on $F$ are equivalent:

(i) $F$ is a $\left(C_{r}\right)$-space;

(ii) $F$ is $\left(B_{r}\right)$-complete;

(iii) $(E, F) \in \mathcal{C}$ for all barreled spaces $E$;

(iv) if $\mathrm{J}_{1} \subseteq \mathrm{J}$, and $\left(F, \mathrm{~J}_{1}\right)$ is barreled, then $\mathrm{J}_{1}=\mathrm{J}$.

Proof. (a) is due to Mahowald. For a proof, see $[7$, IV, 8.6]. (b) We have already noted the equivalence of (i) and (ii), and we have shown that (i) implies (iii). To see that (iii) implies (iv), note that the identity map from $\left(F, J_{1}\right)$ to $(F, J)$ is closed and so must be continuous. It remains to be shown that (iv) implies (i). Let $D$ be a dense linear subspace of $F_{\sigma}^{\prime}$ such that $D \cap B$ is $\sigma$-closed in $B$ for each bounded subset $B$ of $F_{\sigma}^{\prime} . F_{\sigma}^{\prime}$ is quasi-complete, so $D$ is quasi-complete under $\sigma(D, F)$. (Note that $\langle F, D\rangle$ is a dual pair because $D$ is dense in $F_{\sigma}^{\prime}$.) So $(F, \tau(F, D))$ is barreled. Moreover $\tau(F, D)$ is coarser than $\Im$, so we conclude from (iv) that $\tau(F, D)=\Im$. So $D=F^{\prime}$, and the theorem is proved.

6. We now consider the duals under their Mackey topologies (rather than their weak topologies). The difficulty comes in showing that $T^{\prime}$ is continuous in this case. We will prove a general theorem and use it to prove part (iii) of Theorem 1 .

THEOREM 3. Let $T$ be a closed linear map of a Mackey space E into a l.c.s. F. Suppose that $D\left(T^{\prime}\right)$ is a Mackey space under the topology induced from $F_{\tau}^{\prime}$. Then $T$ is continuous if one of the following conditions is satisfied:

(a) $E_{\tau}^{\prime}$ is complete;

(b) $E_{\tau}^{\prime}$ is quasi-complete and $F$ is a $\left(C_{r}\right)$-space;

(c) $E_{\tau}^{\prime}$ is sequentially complete and $F_{\tau}^{\prime}$ is metrizable. 
Proof. $T^{\prime}$ is a closed continuous linear map from $F_{\sigma}^{\prime}$ to $E_{\sigma}^{\prime}$. Let $D=D\left(T^{\prime}\right)$. Then $\langle F, D\rangle$ is a pairing, and the topology $\sigma(D, F)$ on $D$ is identical with the topology induced from $F_{\sigma}^{\prime}$. Now $\tau(D, F)$ is generally finer than the topology induced from $F_{\tau}^{\prime}$, but since this topology is Mackey, the two must be identical. We know that $T^{\prime}$ is a continuous map from $(D, \sigma(D, F))$ to $E_{\sigma}^{\prime}$, so it is continuous as a map from $(D, \tau(D, F))$ to $E_{\tau}^{\prime}[7, I V, 7.4]$, and hence continuous from $F_{\tau}^{\prime}$ to $E_{\tau}^{\prime}$. Apply Lemma 2 to deduce that $D=F^{\prime}$, and Lemma 1 to conclude that $T$ is continuous.

Proof of (iii). If $F_{\tau}^{\prime}$ is metrizable, then every subspace (under the induced topology) is metrizable and hence Mackey. So the result is an immediate corollary of Theorem 3(c).

We remark that a barreled space is a Mackey space and has a quasi-complete Mackey dual. However, a Mackey space $E$ with quasi-complete Mackey dual need not be barreled. For example, let $E=G_{\tau}^{\prime}$, where $G$ is a nonreflexive Fréchet space. So part (iii) of Theorem 1 is neither weaker nor stronger than part (i).

7. We now consider part (iv) of Theorem 1. We define a l.c.s. $(E, J)$ to be ultra-bornological if $J$ is the inductive topology with respect to a family $\left(E_{\alpha}, g_{\alpha}\right)$ where the $E_{\alpha}$ are Banach spaces and the $g_{\alpha}$ are linear maps of $E_{\alpha}$ into $E$. (We do not require that $E$ be an inductive limit.) An ultra-bornological space is both bornological and barreled (for both of these properties are preserved under the formation of inductive topologies). A sequentially complete bornological space is ultrabornological.

A 1.c.s. $F$ is defined by L. Schwartz [8] to be suslinian if there exists a complete separable metrizable space $G$ and a continuous map of $G$ onto $F$. (This definition differs from that of Bourbaki, for $F$ is not required to be metrizable.) Schwartz notes that the distribution spaces listed in [8] are all suslinian. They are also reflexive, so they satisfy the conditions on $F$ in part (v) of Theorem 1 as well as in part (iv).

Proof of (iv). ( $\alpha$ ) is an immediate corollary of Theorem 1(i). For a proof of $(\beta)$, see [8] or [5]. For an outline of the proof of $(\gamma)$, see [3, problem 19B]. If $F$ is an $(L F)$-space, then a more direct proof can be found in [1, Introduction, Theorem B].

Before proving parts (v), (vi) and (vii) of Theorem 1, we prove the following more general theorem. If $E$ is a l.c.s., then let $E_{b}$ denote the associated bornological space. (For definition, see $[7$, p. 63].)

Theorem 4. Let $E$ be a Mackey space such that $E_{b}$ is ultra-bornologi- 
cal. Let $F$ be a semireflexive l.c.s. Then $(E, F) \in \mathcal{C}$ if one of the following three conditions is satisfied.

(a) $E_{\beta}^{\prime}$ is complete, and $F$ satisfies $(\alpha),(\beta)$ or $(\gamma)$ of Theorem 1 (iv);

(b) $\left(E^{\prime}, J\right)$ is quasi-complete for some $J$ such that $\sigma \subseteq J \subseteq \beta$ and $F$ is $a\left(C_{r}\right)$-space;

(c) $\left(E^{\prime}, J\right)$ is sequentially complete for some $J$ such that $\sigma \subseteq \Im \subseteq \beta$, and $F_{\beta}^{\prime}$ is metrizable.

Proof. Let $T$ be a closed linear map of $E$ into $F$. Since $E_{b}$ has a finer topology than $E, T$ is closed as a map from $E_{b}$ to $F$. So, by Theorem 1 (iv), $T$ is continuous under these topologies, and hence maps bounded subsets of $E_{b}$ into bounded subsets of $F$. But the bounded subsets of $E_{b}$ are precisely the bounded subsets of $E$. So $T$ maps bounded subsets of $E$ into bounded subsets of $F$. By taking polars we deduce that $T^{\prime}$ is continuous as a map from $F_{\beta}^{\prime}$ to $E_{\beta}^{\prime}$ (and hence from $F_{\beta}^{\prime}$ to $\left(E^{\prime}, \Im\right)$ ). Also $T^{\prime}$ is closed for these topologies. To see that $D\left(T^{\prime}\right)$ is dense in $F_{\beta}^{\prime}$ we note that $D\left(T^{\prime}\right)$ is dense in $F_{\beta}^{\prime}$, and, by the semireflexivity of $F$, the spaces $F_{\beta}^{\prime}$ and $F_{r}^{\prime}$ are identical. It follows from Lemma 2 that $D\left(T^{\prime}\right)=F^{\prime}$ and so, by Lemma $1, T$ is continuous.

ProOf OF (v), (vi) AND (vii). We will prove that if $E$ is a sequentially complete l.c.s., then $E_{b}$ is ultra-bornological. The result will then be an immediate consequence of Theorem 4.

Let $B$ be the family of closed convex circled bounded nonempty subsets of $E$. For $B \in Q$, let $E_{B}=\bigcup_{n \in N} n B$. Then $E_{B}$ is a normed space under the norm $p_{B}$ where $p_{B}$ is the gauge function of $B$ in $E_{B}$. Moreover, $\left(E_{B}, p_{B}\right)$ is a Banach space. (This is proved in $[7$, p. 63] for the case when $E$ is quasi-complete. The proof for the case of a sequentially complete space $E$ is obtained by adapting that proof.) Now the topology on $E_{b}$ is the inductive topology with respect to the family $\left\{\left(E_{B}, p_{B}, g_{B}\right) \mid B \in Q\right\}$ where $g_{B}$ is the imbedding of $E_{B}$ into $E$. So the theorem is proved.

We remark that parts (v), (vi) and (vii) of Theorem 1 are not included in result (iv). For if $G$ is a nonreflexive Fréchet space, then $G_{\tau}^{\prime}$ is an example of a 1.c.s. that is not ultra-bornological, but that is a sequentially complete Mackey space with complete strong dual.

8. Proof of (viii). Since $F_{\sigma}$ is complete, it follows (by [7], Chapter IV, exercise 6$)$ that $\tau\left(F^{\prime}, F\right)$ is the finest locally convex topology on $F^{\prime}$. So (by [7], Chapter II, exercise 7) every linear subspace of $F_{r}^{\prime}$ is closed. Now let $T$ be a closed linear map of $E$ into $F$. Then $D\left(T^{\prime}\right)$ is both closed and dense in $F_{\tau}^{\prime}$, and so $D\left(T^{\prime}\right)=F^{\prime}$. Hence, by Lemma 1 , $T$ is continuous. 


\section{REFERENCES}

1. A. Grothendieck, Produits tensoriels topologiques et espaces nucléaires, Mem. Amer. Math. Soc. No. 16 (1955).

2. T. Husain, The open mapping and closed graph theorems in topological vector spaces, Clarendon Press, Oxford, 1965.

3. J. L. Kelley, I. Namioka et al., Linear topological spaces, Van Nostrand, Princeton, N. J., 1963.

4. G. Köthe, Topologische Lineare Räume. I, Springer-Verlag, Berlin, 1960.

5. A. Martineau, Sur le théorème du graphe fermé, C. R. Acad. Sci. Paris 263 (1966), 870-871.

6. V. Ptak, Completeness and the open mapping theorem, Bull. Soc. Math. France 86 (1958), 41-74.

7. H. H. Schaefer, Topological vector spaces, Macmillan, New York, 1966.

8. L. Schwartz, Sur le thêrème du graphe fermé, C. R. Acad. Sci. Paris 263 (1966), 602-605.

9. A. Grothendieck, Sur les espaces $(F)$ et $(D F)$, Summa Brasil. Math. 3 (1954), 57-123.

INSTITUTE FOR ADVANCED STUDY 